\title{
Quelques Fourmis des Indes, du Japon et d'Afrique
}

\author{
PAR
}

\section{A. FOREL}

Avec 1 figure dans le texte.

M. le Dr Hons m'a envoyé les Fourmis suivantes du Musée entomologique allemand. Ce sont d'abord ses propres récoltes à Ceylan, dont la partie principale a déjà été décrite par M. le prof. Emery en 1901. Je me contenterai d'énumérer ou de décrire ici les formes qui font défaut dans son travail. En second lieu, j'ai pu prouver que des Fourmis d'origine douteuse provenaient de la province d'Assam (Inde). De ce fait M. Hons a pu, de son còté, conclure qu'il s'agissait de Fourmis reçues de feu le $D^{r}$ Kratz et provenant, on peut dire certainement, de Khasia Hills, à Assam. Enfin, les Fourmis d'Afrique proviennent de M. Conradt, qui les a récoltées à Kamerun. Ici encore, je me contenterai d'énumérer les espèces qui n’ont pas été examinées par MM. Emery et Stit\%.

\section{Ceylan, Assam, Japon, Bombay, Timor et Borneo.}

Odontomachus monticola Em.

†. Khasia Hills (Assam).

Rev. Suisse de Zool. T. 21. 1913. 
Harpegnathos saltator $\mathrm{Sm}$.

‘ $\bigcirc \sigma^{x}$. Khasia Hills (Assam).

Il s'agit évidemment ici d'une mème fourmilière de cette singulière espèce, qui probablement fait les mèmes bonds prodigieux que sa voisine, le cruentatus. Le $\sigma^{T}$ est parfaitement identique à celui que j'ai déjà décrit. Comme lui, il a le thorax noir et la tète et l'abdomen plus ou moins rouges ou jaunâtres. Les ailes sont identiques à celles du cruentatus, avec 3 cellules cubitales distinctes, mais sans le commencement de partage de la première qu'on voit chez le cruentalus, et brunâtres, avec la tache marginale et les nervures brunes. Il ne s'agit donc pas d'ailes anormales, comme l'a cru Emery (voir fig. 1).



FIG. 1. - Harpegnathos venator Sm. ơ.

Diacamma assamense For.

ॐ̆. Khasia Hills (Assam).

Centromyrmex Feae v. ceylonica For.

守. Negombo, Ceylan (Hors).

Rhytidoponera Hanieli n. sp.

守. L. $8^{\mathrm{mm}}, 5$. Mandibules densément et assez finement striées, avec un éclat soyeux, des points épars peu apparents et de nombreuses petites dents. La tète est très large, au moins 1 1/2 fois plus large que le thorax, presque rectangulaire, un peu plus longue que large, légèrement plus large devant que derrière, avec le bord postérieur et les còtés presque droits et avec une légère dépression transversale peu apparente sur le dos de l'occiput. Bord antérieur de l'épistome avancé en triangle obtus. Epistome sans carène. Arètes frontales avec un lobe antérieur 
subhorizontal, presque parallèles en arrière. Les yeux sont situés un peu en arrière du milieu et médiocrement convexes. Le scape dépasse le bord postérieur d'un tiers de sa longueur. Premier article du funicule bien plus court que les suivants, qui sont un peu renflés à l'extrémitéet au moins deux fois plus longs que larges. Le bord antérieur de la tète dépasse sensiblement de còté l'insertion des mandibules. Suture promésonotale profonde, échancrant un peu le profil du thorax. Une dent triangulaire en bas du pronotum. Suture mésoépinotale indistincte. Pas trace de dents à l'épinotum, qui est faiblement convexe. Nœud analogue à celui de l'araneoides Le Guill. r. strigosa Em., mais moins long et avec une surface supérieure moins marquée, légèrement convexe. Le nœud n'est pas plus long que sa hauteur postérieure.

Grossièrement et régulièrement réticulée, avec le fond des mailles luisant. Sur les joues et sur l'épistome il y a, en outre, quelques rides longitudinales entre les réticulations. Abdomen et membrès densément et pas très finement striés en long, sans réticulations, avec des points épars effacés et un éclat soyeux. Tout le corps et les membres pourvus de poils dressés courts, roussàtres, assez raides et obtus, médiocrement abondants. Pubescence à peu près nulle, brune. Mandibules et membres d'un roux brunàtre.

Timor, récolté par M. Haniel; une seule $\Varangle$.

Cette espèce diffère de l'araneoides r. strigosa par sa sculpture, sa large tête et ses yeux moins convexes. A còté des arètes frontales, il y a un petit espace à sculpture plus fine, qui ne forme du reste pas de scrobe proprement dit. A certains égards, cette espèce ressemble un peu à la $R$. (Chalcoponera) obscurum For.

Leptogenys (Lobopelta) diminuta Sm. sens strict.

ఢ. Khasia Hills (Assam).

Leplogenys (Lobopelta) diminuta Sm. r. sarasinorum For. v. nitidiceps Em.

๖. Bandarawella, Ceylan (Hors). 
Leptogenys (Lobopelta) Kilteli Mayr.

ॐ. Khasia Hills (Assam).

Sima thagatensis For.

ๆ. Khasia Hills (Assam).

Pheidole megacephala F.

‡ 2 . Puttalam et Bandarawella, Ceylan (Hors).

Pheidole rhominoda Mayr. r. taprobanae For.

ґ. Puttalam, Ceylan (Hors).

Cremastogasier Rothneyi Mayr. v. civa For.

兰. Bandarawella, Ceylan (Horx).

Cremastogaster subnuda Mayr.

ґ. Trincomali, Ceylan (Hons).

Cremastogaster Rogenhoferi Mayr.

‘. Khasia Hills (Assam).

Cremastogaster (Oxygyne) aberrans For. v. Assmuthi n. v.

$\Varangle$ L. $3^{\mathrm{mm}}, 6$ à $3^{\mathrm{mm}}, 7$. Entièrement noir ou d'un noir à peine brunâtre. Epines de l'épinotum distinctement plus longues que chez le type. Le premier nœud du pédicule est plus court et plus large, plus large que long (un peu plus long que large chez le type), presque aussi large que le second nœud. La tête est aussi un peu plus convexe et un peu moins large derrière. Les membres sont bruns, les mandibules seules rougeâtres.

La $\subsetneq$ est absolument identique à celle du type de l'espèce par sa forme, sa sculpture, sa taille et tout le reste.

Bombay, récoltées par M. Assmuth et reçues par M. Wasmann.

Pheidologeton affinis Jerd. v. spinosior For.

ґ. Nalanda, Ceylan (Hors).

Cataulacus granulatus Latr.

ॐ. Khasia Hills (Assam). 
Technomyrmex albipes Sm. r. Wedda n. st.

ఢ. L. $2^{\mathrm{mm}}, 2$ a $2^{\mathrm{mm}}, 6$. Tout le corps d'un roussâtre un peu brun, avec les membres légèrement plus clairs et les tarses un peu plus jaunàtres. Tout l'Insecte est un peu moins trapu que l'espèce typique; la tète surtout est plus étroite, bien plus longue que large, aussi étroite derrière que devant, avec le bord postérieur à peu près droit et les yeux légèrement plus petits. La face basale de l'épinotum est un peu moins courte et sa face déclive légèrement plus convexe. Les scapes sont d'une idée plus longs. La pubescence est plus faible, mais la sculpture est la mème.

$\subsetneq$ L. $3^{\mathrm{mm}}, 1$. Mêmes différences que pour l'ouvrière. Les ailes manquent. Cette race a à peu près la couleur de la r. brunneus For., mais elle s'en distingue par sa tète étroite, sa faible pubescence et ses scapes plus longs.

Bandarawella et Nalanda Ceylan (Hors).

Tapinoma indicum For.

ॐ. Trincomali, Ceylan (Новм).

Plagiolepis Wroughtoni For.

ґ. Bandarawella, Ceylan (Hors).

Acropyga acutiventris Rog.

ґ. Weligama, Ceylan (Hors).

Une variété un peu plus petite, avec les yeux relativement plus petits, se rapprochant un peu de la r. australis For. A ce propos je dois dire que je ne puis confirmer ce que disent Mayr et Emery sur les antennes de cette espèce; chez tous mes nombreux exemplaires, les scapes de l'acutiventris dépassent distinctement le bord postérieur de la tète de bien plus de leur épaisseur, et les articles du funicule, du moins ceux de la base, sont plus longs que larges.

Prenolepis Taylori For.

․ Trincomali, Ceylan (Hors).

Camponotus fallax Nyl. v. Keihitoi n. v.

广. L. $5^{\mathrm{mm}}, 5$ à $6^{\mathrm{mm}}$. Entièrement noire, avec les membres d'un 
brun rougeàtre, les mandibules, le bord antérieur de la tête et les tarses rougeâtres. Le bord postérieur des segments de l'abdomen est d'un jaune doré. Cette variété diffère de la v. Bruni par son écaille mince et de toutes les autres par son épistome subcaréné, avec un lobe antérieur assez avancé et seulement une échancrure très faible à son bord antérieur. Ses mandibules à 5 dents et tout le reste le rapportent à part cela au fallax.

† L. $9^{\mathrm{mm}}$. Exactement les mêmes différences que pour l'ouvrière. Echancrure de l'épistome un peu plus nette. Ailes assez fortement enfumées de brun, avec la tache marginale brune.

Ile Kondo, prov. Nagato (Japon). Récolté par M. Nawa et reçu de M. le prof. Iто.

Camponotus (Myrmoturba) maculatus F. r. mitis Sm. v. Bacchus $\mathrm{Sm}$.

古 $\sigma^{x}$. Nalanda, Ceylan (Hors).

Camponotus (Orthonotomyrmex) sericeus F. r. peguensis Em. $\mathrm{v}$ igniceps $\mathrm{n}$. v.

ఢ. Je donne ce nom à la variété du peguensis qui a la tête et les antennes rouges chez la $q$ et l'ouvrière, tandis que le reste $\mathrm{du}$ corps est noir. La r. peguensis Em. a la pubescence et la forme générale du sericeus typique et non les caractères de la r. opaciventris Mayr.

Matale, Kandy et Pukalam Ceylan (Hors) ( $q$ q).

Polyrhachis proxima Rog.

ґ. Khasia Hills (Assam).

Polyrhachis acantha Sm. r. timorensis n. st.

భ. L. $7^{\mathrm{mm}}, 5$. Beaucoup plus grande que le type de l'espèce et voisine surtout de la v. argentea Mayr. Les yeux sont beaucoup moins convexes, sans ètre pourtant entièrement plats. Le bord postérieur de la tête est bien plus convexe et la tête relativement moins large, plus longue que large. Le pronotum a la même forme fortement convexe, mais ses épines antérieures sont bien plus longues, plis aiguës et assez fortement courbées à l'extrémité. 
Les épines de l'épinotum sont très longues, très épaisses à leur base, très divergentes, aussi longues que la face basale de l'épinotum et le mésonotum pris ensemble. Les épines de l'écaille sont très longues et très aiguës aussi, aussi longues que celles de l'épinotum, mais moins courbées et moins horizontales que chez l'argentea, dirigées à la fois en haut et en dedans. Les deux petites dents médianes sont comme chez l'argentea. La sculpture et la pubescence sont comme chez l'argentea, mais la pubescence est beaucoup plus faible sur l'abdomen, qui est aussi plus faiblement réticulé, assez luisant. La couleur est comme chez l'argentea, mais l'abdomen et les pattes sont d'un roux jaunàtre avec les tarses bruns.

Timor (Haniel), un seul exemplaire.

Polyrhachis cephalotes Em.

$\subsetneq \sigma^{x}$. Balik Papan, Bornéo. Récolté avec le nid par M. KaмpMEINERT.

ㅇ (non encore décrite). L. $10^{\mathrm{mm}}, 5$. La iête n'est pas beaucoup plus étroite que le large thorax. Les ailes sont brunâtres, les tarses et les tibias jaunâtres. Du reste comme l'ouvrière.

$\sigma^{\Upsilon} \mathrm{L} \cdot 7^{\mathrm{mm}}$. Tête épaisse, à bord postérieur assez droit, peu convexe. Pronotum très convexe. Epinotum convexe, sans dents ni tubercules. Ecaille épaisse, échancrée au sommet. Tète assez mate, densément réticulée; le reste plutòt subopaque, plus faiblement réticulé. Pubescence jaunâtre, nette, mais peu dense, ne cachant nullement la sculpture. D'un noir brunàtre. Funicules, cuisses, hanches et bord des segments abdominaux brunâtres. Une bande longitudinale de chaque còté du mésonotum, les scapes, les mandibules, les tibias et les tarses d'un jaune roussâtre. Ailes brunàtres.

Le nid qui se trouvait avec ces Insectes est fait d'un papier brunâtre, solide et épais, et paraît contenir des cases et galeries assez nombreuses.

Polyrhachis convexa Rog.

家. Timor (Haniel). 


\section{Afrique.}

Paltothyreus tarsalus $\mathrm{F}$.

并 $\sigma^{x}$. Kamerun (Conradt).

Plectroctena mandibularis $\mathrm{Sm}$.

家. Kamerun (Conradt).

Odontomachus assiniensis Em.

家 $ᄋ \sigma^{x}$.

† L. $15^{\mathrm{mm}}$. Plus foncée que l'ouvrière; d'un brun foncé, avec l'abdomen presque noir, les pattes, les mandibules et les antennes roussâtres. Tout le reste est exactement comme chez l'ouvrière, mais la sculpture est plus grossière. Mésonotum strié en long, pronotum strié en travers, scutellum lisse comme l'abdomen; les ailes manquent.

$\sigma^{r} \mathrm{~L} .7^{\mathrm{mm}}, 2$. Entièrement d'un jaune à peine roussâtre, avec les funicules, sauf leur premier article, brunâtres et les ailes brunâtres aussi. Les yeux sont en forme de haricot et la tête assez longue est très fortement convexe derrière. Ocelles bien plus grands que leurs intervalles. Pédicule avec une forte dent en dessous; il est en còne fort obtus, arrondi au sommet. Assez luisant et irrégulièrement ponctué avec des rides çà et là. Pattes sans poils dressés; ces derniers fort épars sur le corps. Le $\sigma^{\Upsilon}$ et les $\Varangle$ proviennent de Kamerun (Conradt); la $q$ d'Amani, en Afrique orientale, récoltée par M. Zimaer. Je la possédais depuis longtemps sans l'avoir décrite.

\section{Leptogenys Conradti $\mathrm{n}$. sp.}

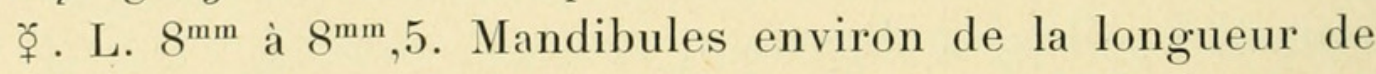
la tête, courbées à la base et à la dent terminale, presque droites dans l'entre-deux, également étroites de la base à l'extrémité, sans dents sauf la terminale, lisses, luisantes, avec d'assez gros points fort épars. Epistome caréné, avancé en lobe triangulaire. Tête en trapèze, presque $1^{1 / 2}$ fois plus large devant que derrière, avec les còtés presque droits, ainsi 
que le bord postérieur qui est constitué presque en entier par le bord articulaire. Le bord postérieur des yeux, qui sont assez convexes, atteint le milieu de la tète, et leur bord antérieur est un peu plus près du bord antérieur de la tète que la longueur de leur diamètre. Le scape dépasse le bord postérieur de la tête de plus d'un tiers de sa longueur, et le second article du funicule est au moins $3^{1 / 2}$ fois plus long que large. Thorax peu convexe, sans trace de dents. Nœud médiocrement élargi derrière, 1 1/2 fois plus long que large, armé devant (en bas) de deux dents triangulaires, dessous (en avant) d'un lobe comprimé, un peu dentiforme devant, enfin prolongé au sommet par une forte dent unique, dirigée en arrière et en haut, deux fois plus longue que large, et un peu obtuse à l'extrémité. Derrière cette dent, le nœud est perpendiculairement tronqué et subbordé. En arrière il est aussi haut que long, mais il s'abaisse en avant par une courbe arrondie. Abdomen médiocrement échancré derrière le postpétiole, qui est aussi large, mais plus court que le segment suivant.

Luisante, très grossièrement rugueuse sur la tête, le thorax et le nœud. Les rugosités sont fort irrégulières, en bonne partie réticulaires, avec une direction plus ou moins longitudinale, surtout sur la tête. Postpétiole, abdomen et membres lisses et luisants avec des points épars. Tout le corps, ainsi que les membres, assez abondamment couverts d'une pilosité assez brillante et assez longue, d'un jaune très clair. Pubescence presque nulle. Pattes, scapes et mandibules d'un brun un peu roussâtre. Tarses et funicules plutòt d'un roux brunâtre; tout le reste noir.

Kamerun (Conradt). Cette espèce est très remarquable, tant par sa sculpture que par la pointe de son pétiole.

\section{Cerapachys cribinodis Em.}

భ. Johann-Albrechtshöhe, Nord-Kamerun (Conidot).

Dorylus (Anomma) nigricans Illig. v. funerea Em.

$\sigma^{\Upsilon}$. Kamerun (Connadt). 
Dorylus depilis Em.

$\sigma^{x}$. Kamerun (Conradt).

Dorylus politus Em.

ґ. Johann-Albrechtshöhe, Nord-Kamerun (Conradt).

Sima (Pachysima) Aethiops Sm.

๘. Kamerun (Conradt).

Cataulacus erinaceus Stitz.

ґ. Kamerun (Conradt).

Cataulacus guineensis Sm. v. alenensis Stitz.

广. Kamerun (Conradt).

Triglyphothrix gabonensis André.

ґ. Johann-Albrechtshöhe, Nord-Kamerun (Conradt).

Tetramorium aculeatum Mayr.

ґ . Kamerun (Conradt).

Pheidole speculifera Em.

‘. Johann-Albrechtshöhe, Nord-Kamerun (Conpadt). Variété un peu plus claire et un peu plus lisse.

Cremastogaster (Atopogyne) Luciae For.

ㅇ. Kamerun (Conradt).

Cremastogaster (Atopogyne) Homeri n. sp.

․ L. $5^{\mathrm{mm}}, 4$. Mandibules fortement striées et ponctuées, armées de 5 dents. Bord antérieur de l'épistome légèrement avancé en arc et légèrement déprimé transversalement. Tête de $1 / 5$ à $1 / 4$ plus longue que large, rectangulaire, aussi large devant que derrière, à còtés et à bord postérieur droits. Les yeux sont situés presque au milieu (à peine en avant). Les scapes atteignent le quart postérieur de la tête. Les articles 2 à $7 \mathrm{du}$ funicule sont beaucoup plus épais que longs les articles 2 et 3 presque deux fois). Massue de 3 articles. Thorax beaucoup plus étroit que la tête, avec une face déclive assez abrupte de l'épinotum et presque sans face basale. Premier nœud du pédi- 
cule en trapèze renversé, un peu plus large devant que derrière. Second nœud sans trace de sillon médian, un peu plus large que long, et légèrement plus large que le devant du premier nœud.

Lisse et luisant, même les joues, sauf tout près du bord antérieur, où elles ont quelques stries longitudinales. Tête avec des points épars, mais réguliers, pas très profonds, ni gros, mais fort apparents. Ponctuation plus faible sur le reste du corps. Les pattes ont des poils courts et obliques, qui sont presque adjacents sur les scapes. Pilosité dressée, courte et fortiéparse, jaunàtre sur le corps. Pubescence fort éparse aussi. D'un roux plus ou moins jaunâtre ou brunâtre, avec les ${ }^{2}{ }_{3}$ postérieurs de l'abdomen bruns. Mandibules roussàtres avec les dents brunes. Membres d'un jaune un peu roussâtre. Ailes subhyalines, faiblement jaunàtres, avec les nervures et la tache marginale fort pâles.

Johann-Albrechtshöhe, Nord-Kamerun (ConradT).

Cremastogaster chlorotica Em.

ґ. Johann-Albrechtshöhe, Nord-Kamerun (Conradt). Correspond exactement au type d'Euery, mais il porte la même étiquette avec la même provenance que l'espèce précédente. Des $q$ avec l'étiquette: “Kamerun. Conrad » correspondent par contre a la $q$ décrite avec certains doutes par Emery comme étant celle du chlorotica. Il n'est pas impossible que l'espèce précédente (Homeri) soit celle du chlorotica, mais la chose étant absolument douteuse, j’ai préféré ne rien changer et donner un nom nouveau; l'avenir éclaircira la question.

\section{Cremastogaster pauciseta Em.}

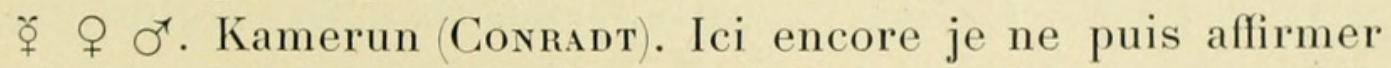
que le petit $\sigma^{x}$ brun, aux ailes hyalines, long de $3^{\mathrm{mm}}$, soit vraiment celui de cette espèce.

Cremastogaster Buchneri For.

․ Kamerun (Conradt). Correspond bien à la description d'Emery. 
Cremastogusler Buchneri For. r. Winkleri For.

ॐ. Johann-Albrechtshöhe, Kamerun (Conradt).

Cremastogaster (Oxygyne) Stadelmanni Mayr.

. Kamerun (Conradt).

Oecophylla smaragdina F. v. longinoda Latr.

q $\sigma^{T}$. Kamerun (Conradt).

Camponotus (Myrmoturba) maculatus Fabr. r. guttatus Em.

$\ddot{q} \widetilde{\sigma}^{T}$. Kamerun (Conradt). Emery a bien décrit l'ouvrière et la $q$, mais pas le $\sigma^{x}$.

$\sigma^{\Upsilon}$. L. $7^{\mathrm{mm}}, 8$. Assez luisant. Tête et thorax, en particulier le mésonotum, assez densément réticulés. Abdomen et épinotum fort luisants et très faiblement chagrinés. Ecaille épaisse, largement échancrée au sommet. Pilosité très éparse, assez longue, jaune pâle. D’un jaune à peine roussàtre, uniforme sur le corps et les membres. Ailes faiblement jaunâtres avec la tache et les nervures jaunàtres. Du reste comme l'ouvrière.

Camponotus (Orthonomyrmex) Meinerti For.

○. Kamerun (Conradt).

Camponotus (Orthonotomyrmex) Meinerli For. r. reginae For. 家. Kamerun (Conradt).

Camponotus (Orthonomyrmex) chrysurus Gerst. r. acustisquamis Mayr.

隹. Kamerun (COnRadt).

१. L. $12^{\mathrm{mm}}, 5$. Comme l'ouvrière et avec la mème écaille élevée et tranchante. Mais l'épistome a au milieu un sillon longitudinal, comme chez l'espèce typique. La tête est assez rectangulaire, beaucoup plus longue que large, peu rétrécie devant et très faiblement échancrée derrière, presque droite. Elle est beaucoup plus large que le thorax. Du reste comme l'ouvrière, avec les ailes enfumées de brunàtre. 
Camponotus (Myrmotrema) foraminosus For. sens. strict.

q (non encore décrite). L. $9^{\mathrm{mm}}, 8$. Tout à fait semblable à l'ouvrière. Les fossettes sont très abondantes et très distinctes sur l'épistome, les joues et les còtés de la tête, un peu plus superficielles sur le front et le vertex, s'évanouissant sur l'occiput. La tête est un peu plus large que le thorax. L'écaille est biconvexe (plus convexe devant) et assez épaisse. La pubescence est faible sur la tête, abondante déjà sur le thorax, qu'elle recouvre d'un duvet grisâtre, mais surtout abondante et épaisse sur l'abdomen, où elle est un peu jaunàtre et à la fois beaucoup plus courte et beaucoup plus dense que chez la r. chrysogaster Em. Il s'ensuit qu'on a peine à voir la sculpture, qui est assez densément réticulée-ponctuée. La pilosité dressée est jaunâtre, assez longue et fort éparse. Elle est assez pointue, sauf quelques soies un peu plus épaisses et obtuses sur l'écaille, le devant de l'abdomen et l'épistome. Les pattes n'ont pas de poils dressés, seulement une pubescence adjacente et éparse. Noire. Extrémité des tarses, antennes, mandibules et devant des joues d'un roux un peu brunâtre. Ailes enfumées de brunâtre, avec les nervures jaunâtres et la tache marginale brune.

Kamerun (Conratot).

Camponotus (Phasmomyrmex) Buchneri. For.

¡. Kamerun (Conradt).

Camponotus (Myrmamblys) Ferreri For. n. sp.

ๆ. L. $7^{\mathrm{mm}}, 2$. Mandibules épaisses, armées de 6 dents, subopaques, assez densément réticulées-ponctuées, avec de gros points espacés, plus petits vers la base. Leur bord externe est droit. Tête distinctement plus longue que large et plus étroite devant que derrière, avec le bord postérieur faiblement convexe; les còtés sont assez convexes derrière les yeux, mais presque droits devant. Epistome sans carène distincte, bi-échancré, très faiblement avancé en arc devant, avec d'étroites portions latérales, distinctement plus large devant que derrière, mais plus haut que son bord postérieur (aussi haut que sa portion médiane antérieure). Aire frontale peu distincte, assez transversale, 
beaucoup plus large que longue. Arètes frontales sigmoïdes, assez fortement divergentes derrière, où elles sont au moins aussi éloignées l'une de l'autre que des còtés de la tète. Celle-ci est fort épaisse et convexe, avec une apparence subtronquée devant, à partir de l'articulation des antennes. Les yeux sont très grands, occupant un bon tiers des còtés. Le scape dépasse le bord postérieur d'au moins deux fois son épaisseur. Thorax un peu plus étroit que la tête. Face basale de l'épinotum séparée du postscutellum par une échancrure, après laquelle elle remonte un peu; puis elle est fortement convexe avant d'arriver à la face déclive, qui est droite de profil, très abrupte et presque deux fois plus longue que la face basale. Ecaille un peu cunéiforme, assez large à la base et tranchante au sommet, faiblement convexe devant, droite derrière, où elle est mème un peu concave et latéralement subbordée, à bord supérieur droit. Cuisses légèrement dilatées et comprimées, sans petits piquants.

Epistome et joues à peu près mats, densément réticulésponctués, avec quelques grosses fossettes assez effacées. Le reste de la tète et du thorax assez fortement réticulé et assez luisant. Abdomen très luisant et faiblement chagriné. Sauf les tibias, qui n'ont qu'une pubescence jaunàtre à peine soulevée, le corps et les membres (aussi les scapes) sont recouverts d'une pilosité dressée, jaunàtre, irrégulière et assez courte. Sur le devant de la tète cette pilosité est bien plus abondante, fort courte et obtuse a l'extrémité. D'un brun assez foncé. Front, épistome, joues, mandibules, antennes et pattes jaunes. Bord postérieur des segments abdominaux d'un jaunâtre sale. Ailes jaunàtres, avec les nervures et la tache marginale jaunes.

Kamerun (Conradt). Cette espèce est assez curieuse. Quoique à peine tronquée, la tête, par sa couleur, semble indiquer un genre de vie analogue à celui des Colobopsis ròle de portier chez la $q$ et les grandes $\not{q})$. Cette forme rappelle certaines espèces des Iles de la Sonde. Un seul exemplaire.

Polyrhachis militaris F.

ґ. Kamerun (Conradt). 
Polyrhachis decemdentata André \& (non encore décrite). L. $6^{\mathrm{mm}}, 5$. Très semblable à l'ouvrière, mais plus grossièrement striée en long, surtout sur le thorax, y compris le mésonotum. Les épines du pronotum sont très larges, aussi larges que longues. L'épinotum n'a derrière que deux très petites dents aussi larges que longues. Sa face basale est moins bordée et plus convexe que chez l'ouvrière; le mésonotum et le scutellum ne sont que faiblement subbordés. Les dents médianes de l'écaille sont obtuses et un peu plus larges que longues. Les deux dents, ou plutòt épines moyennes, sont un peu plus courtes que chez l'ouvrière, et enfin les deux dents inférieures sont un peu moins courbées en arrière, triangulaires, aussi larges que longues. Les ailes manquent. L'abdomen est plus fortement réticulé que chez l'ouvrière, subopaque. Tout le reste identique à l'ouvrière.

Kamerun (Conradt).

N.-B. - Dans la "Revue zoologique africaine ", vol. 2, 1913, p. 310, j’ai décrit une Myrmecia piriformis Sm. r. gigas et j’ai dit qu'Emery considérait la spadicea Mayr comme une pseudogyne, parce qu'elle avait un ocelle. M. Emery m'écrit qu'il la considère comme une $q$ ergatogyne, non pour cette raison, mais parce qu'elle a le thorax à forme un peu $q$. Ceci n'est pas du tout le cas chez la gigas, qui a le thorax absolument $\not{q}$. Je tenais simplement à faire cette rectification. 


\section{$2 \mathrm{BHL}$ Biodiversity Heritage Library}

Forel, Auguste. 1913. "Quelques Fourmis des Indes, du Japon et d'Afrique." Revue suisse de zoologie 21, 659-673. https://doi.org/10.5962/bhl.part.37159.

View This Item Online: https://www.biodiversitylibrary.org/item/40668

DOI: https://doi.org/10.5962/bhl.part.37159

Permalink: https://www.biodiversitylibrary.org/partpdf/37159

\section{Holding Institution}

MBLWHOI Library

\section{Sponsored by}

MBLWHOI Library

\section{Copyright \& Reuse}

Copyright Status: NOT_IN_COPYRIGHT

This document was created from content at the Biodiversity Heritage Library, the world's largest open access digital library for biodiversity literature and archives. Visit BHL at https://www.biodiversitylibrary.org. 